\title{
Building informational model management automation
}

\author{
Pavel Chelyshkov ${ }^{*}$ \\ Moscow State University of Civil Engineering, Yaroslavskoe shosse, 26, Moscow, 129337, Russia
}

\begin{abstract}
This paper describes the approaches to analysis in order to optimize the construction facilities (buildings and complexes) information models management. Two criteria have been proposed and discussed for the model quality evaluation: model informational saturation criterion and model topicality criterion. Practical application of the first criterion enables to define the optimal plan of information model filling. Herewith, the optimization is carried out through the maximum volume of useful information. The criterion is used at all stages of the model life cycle during the course of the model data updating. The second criterion allows to evaluate the need in the model data update. Being a vector (in the space of the modelling object significant values) of deviation of the model data from the model object data, the criterion represents the need to initiate the model data updating procedure using the predefined rules
\end{abstract}

\section{Introduction}

Information technologies development trends in general, and information modeling tools in particular have led to the situation, when the use of information modeling tools and technologies in construction and utility complex are not an exception. Buildings and construction facilities information models are created for different stages of the life cycle and for finding various solutions [1-7]. These models are characterized by different structures, scope of information, and access rules.

At the same time, along with the wide use of these technologies [7], there is no generally accepted (both industry, and government level) rules for management of information, development, and use of information models for various stages of the buildings life cycle [7-12]. This situation leads to the use of irrational ways for creation of information models related to entering excessive data, longer time needed for models creation, puzzled structure of the model, etc.

\footnotetext{
* Corresponding author: chelyshkovpd@mgsu.ru
} 


\section{Main part}

The use of information modeling technologies for management tasks in city scale and area scale distributed and grid system imposes the requirements for elaboration of the formalized rules for data exchange and information management. Forming and formalization of the general rules and information management concepts, information models development and use, are related to the need in formalization of the information models measurable quality criteria. Herewith, the criteria reflecting the quality of the information models in most cases are closely linked with the context of use of the specific model, and are subjective, i. e. poorly formalized for the general case.

In terms of structure, the model consists of the aggregation of submodels implementing the functions of data storage, processing, and generation.

The objective quality criterion of the information model calculated with no dependence on the model use context may be the model compilation entropic criterion of efficiency.

Information entropy as a function of the object condition is calculated using the formula (1).

$$
H(E)=-\sum_{i=1}^{l} P_{i} \log _{2} P_{i}
$$

where $H$-entropy; $E_{i}$ - object condition; $P_{i}$ - probability of the object condition $E_{i}$; $l$-count of conditions $E_{i}$.

For $m$-component system (model), with the share of each component within the general scope of the system (model) $c_{i}$, the following expression is valid:

$$
\sum_{i=1}^{m} c_{i}=1
$$

If microscopic volumes are randomly outlined in this system, the probability of having the elements shares in each of these volumes equal to $c_{1}, c_{2}, \ldots, c_{m}$ is for each element:

$$
P_{i}=N_{i} / N=c_{i}
$$

where $N_{i}$-count of elements of $i$-th population in the microscopic volume; $N$-total count of elements in the microscopic volume.

When the physical system conditions count is fixed, its information entropy is maximum when the results of these conditions are equal:

$$
P_{1}=P_{2}=\ldots=P_{n}=1 / N
$$

Then:

$$
H_{\max }=\log _{2} n
$$


Then, using the parameter $\Phi-$ deviation of the system (model) entropy from maximum possible entropy of the system (model), we can judge about the system (model) synthesis method efficiency. The smaller the parameter $\Phi$ is, the more efficient the system (model) synthesis method is.

$$
\Phi=\frac{H_{\max }-H}{H_{\max }}
$$

The most important practical aspect of this approach is the outlining equal microscopic volumes of data in the model. A model is considered that is used throughout the entire life cycle of the construction facilities - from conceptual design to disposal.

Let us consider an exemplary calculation. Let us consider the power supply system general parameters submodel at the design and engineering stage in a simplified manner. Let this submodel contain the data about the power supply sources of the first reliability category and the second reliability category. Let us assume (as an example) that there are the following types of data: P1 - input data about first category power supply sources, P2 input data about second category power supply sources, P3 - internal useful data about first category power supply sources, $\mathrm{P} 4$ - internal useful data about second category power supply sources. The data are distributed as follows: P1 - 65\%, P2 - 10\%, P3 - 20\%, P4$5 \%$.

For 1 option:

$$
H_{\text {max }}=\log _{2} n=\log _{2} 4=2
$$

$$
\begin{gathered}
H(E)=-\sum_{i=1}^{l} P_{i} \log _{2} P_{i}=-\left(0,65 \cdot \log _{2} 0,65+0,1 \cdot \log _{2} 0,1+0,2 \cdot \log _{2} 0,2\right. \\
+\mathbf{0 , 0 5} \cdot \log _{2} \mathbf{0 , 0 5}=\mathbf{1 , 4 1 7} \\
\Phi=\frac{H_{\max }-H(E)}{H_{\max }}=\frac{2-1,417}{2}=0,292
\end{gathered}
$$

For 2 option:

$$
\begin{gathered}
H(E)=-\sum_{i=1}^{l} P_{i} \log _{2} P_{i}=-\left(\mathbf{0 , 6 5} \cdot \log _{2} \mathbf{0 , 6 5}\right)-\left(\mathbf{0 , 1} \cdot \log _{2} \mathbf{0 , 1}+\mathbf{0 , 2} \cdot \log _{2} \mathbf{0 , 2}\right. \\
\left.+\mathbf{0 , 0 5} \cdot \log _{2} \mathbf{0 , 0 5}\right)=\mathbf{1 , 8 0 1} \\
\Phi=\frac{H_{\max }-H(E)}{H_{\max }}=\frac{2-1,801}{2}=0,100
\end{gathered}
$$

For 3 option:

$$
\begin{gathered}
H(E)=-\sum_{i=1}^{l} P_{i} \log _{2} P_{i}=-\mathbf{0 , 2} \cdot \log _{2} 0,2-\left(0,65 \cdot \log _{2} 0,65+0,1 \cdot \log _{2} 0,1\right. \\
+\mathbf{0 , 0 5} \cdot \log _{2} \mathbf{0 , 0 5}=\mathbf{1 , 3 9 7} \\
\Phi=\frac{H_{\max }-H(E)}{H_{\max }}=\frac{2-1,397}{2}=0,302
\end{gathered}
$$


For 4 option:

$$
\begin{gathered}
H(E)=-\sum_{i=1}^{l} P_{i} \log _{2} P_{i}=-\mathbf{0 , 1} \cdot \log _{2} 0,1-\left(0,65 \cdot \log _{2} 0,65+0,2 \cdot \log _{2} 0,2\right. \\
+\mathbf{0 , 0 5} \cdot \log _{2} \mathbf{0 , 0 5}=\mathbf{1 , 3 1 6} \\
\Phi=\frac{H_{\max }-H(E)}{H_{\max }}=\frac{2-1,316}{2}=0,327
\end{gathered}
$$

For 5 option:

$$
\begin{gathered}
H(E)=-\sum_{i=1}^{l} P_{i} \log _{2} P_{i}=-\mathbf{0 , 0 5} \cdot \log _{2} 0,05-\left(0,65 \cdot \log _{2} 0,65+0,1 \cdot \log _{2} 0,1\right. \\
\left.+\mathbf{0 , 2} \cdot \log _{2} \mathbf{0 , 2}\right)=\mathbf{1 , 4 1 7} \\
\Phi=\frac{H_{\max }-H(E)}{H_{\max }}=\frac{2-1,417}{2}=0,292
\end{gathered}
$$

For 6 option:

$$
\begin{gathered}
H(E)=-\sum_{i=\mathbf{1}}^{l} \boldsymbol{P}_{\boldsymbol{i}} \log _{2} \boldsymbol{P}_{\boldsymbol{i}}=-\left(\mathbf{0 , 6 5} \cdot \log _{2} \mathbf{0 , 6 5}+\mathbf{0 , 1} \cdot \log _{2} \mathbf{0 , 1}\right)-\left(\mathbf{0 , 2} \cdot \log _{2} \mathbf{0 , 2}\right. \\
\left.+\mathbf{0 , 0 5} \cdot \log _{2} \mathbf{0 , 0 5}\right)=\mathbf{1 , 7 0 3} \\
\Phi=\frac{H_{\max }-H(E)}{H_{\max }}=\frac{2-1,703}{2}=0,149
\end{gathered}
$$

For 7 option:

$$
\begin{gathered}
H(E)=-\sum_{i=1}^{l} P_{i} \log _{2} P_{i}=-\left(\mathbf{0 , 6 5} \cdot \log _{2} 0,65+\mathbf{0 , 0 5} \cdot \log _{2} 0,05\right)-\left(\mathbf{0 , 1} \cdot \log _{2} \mathbf{0 , 1}\right. \\
+\mathbf{0 , 2} \cdot \log _{2} \mathbf{0 , 2}=\mathbf{1 , 2 8 1} \\
\Phi=\frac{H_{\max }-H(E)}{H_{\max }}=\frac{2-1,281}{2}=0,360
\end{gathered}
$$

For 8 option:

$$
\begin{gathered}
H(E)=-\sum_{i=1}^{l} P_{i} \log _{2} P_{i}=-\left(0,65 \cdot \log _{2} 0,65+0,2 \cdot \log _{2} 0,2\right)-\left(0,1 \cdot \log _{2} 0,1\right. \\
\left.+\mathbf{0}, \mathbf{0 5} \cdot \log _{2} \mathbf{0 , 0 5}\right)=\mathbf{1}, 279 \\
\Phi=\frac{H_{\max }-H(E)}{H_{\max }}=\frac{2-1,279}{2}=0,361
\end{gathered}
$$

For 9 option:

$$
\begin{gathered}
\left.H(E)=-\sum_{i=\mathbf{1}}^{l} \boldsymbol{P}_{\boldsymbol{i}} \log _{2} \boldsymbol{P}_{\boldsymbol{i}}=-\left(\mathbf{0 , 6 5} \cdot \log _{2} \mathbf{0 , 6 5 + 0 , 1} \cdot \log _{2} \mathbf{0 , 1}\right)-\mathbf{0 , 2} \cdot \log _{2} \mathbf{0 , 2}\right) \\
-\mathbf{0 , 0 5} \cdot \log _{2} \mathbf{0 , 0 5}=\mathbf{1 , 1 3 4} \\
\Phi=\frac{H_{\max }-H(E)}{H_{\max }}=\frac{2-1,134}{2}=0,433
\end{gathered}
$$


For 10 option:

$$
\begin{gathered}
\left.H(E)=-\sum_{i=\mathbf{1}}^{l} \boldsymbol{P}_{\boldsymbol{i}} \log _{2} \boldsymbol{P}_{\boldsymbol{i}}=-\left(\mathbf{0 , 6 5} \cdot \log _{\mathbf{2}} \mathbf{0 , 6 5}+\mathbf{0 , 2} \cdot \log _{2} \mathbf{0 , 2}\right)-\mathbf{0 , 1} \cdot \log _{2} \mathbf{0 , 1}\right) \\
\left.-\mathbf{0 , 0 5} \cdot \log _{2} \mathbf{0 , 0 5}\right)=\mathbf{1 , 2 4 6} \\
\Phi=\frac{H_{\max }-H(E)}{H_{\max }}=\frac{2-1,246}{2}=0,377
\end{gathered}
$$

For 11 option:

$$
\begin{gathered}
\left.H(E)=-\sum_{i=1}^{l} P_{i} \log _{2} P_{i}=-\left(\mathbf{0 , 6 5} \cdot \log _{2} 0,65+\mathbf{0 , 0 5} \cdot \log _{2} 0,05\right)-\mathbf{0 , 2} \cdot \log _{2} 0,2\right) \\
\left.-\mathbf{0 , 1} \cdot \log _{2} \mathbf{0 , 1}\right)=\mathbf{1 , 1 9 7} \\
\Phi=\frac{H_{\max }-H(E)}{H_{\max }}=\frac{2-1,197}{2}=0,402
\end{gathered}
$$

For 12 option:

$$
\begin{gathered}
\left.H(E)=-\sum_{i=1}^{l} P_{i} \log _{2} P_{i}=-\left(\mathbf{0 , 6 5} \cdot \log _{2} 0,65+\mathbf{0 , 1} \cdot \log _{2} 0, \mathbf{1}\right)-\mathbf{0 , 0 5} \cdot \log _{2} 0,05\right) \\
-\mathbf{0 , 2} \cdot \log _{2} \mathbf{0 , 2}=\mathbf{1 , 3 5 4} \\
\Phi=\frac{H_{\max }-H(E)}{H_{\max }}=\frac{2-1,354}{2}=0,323
\end{gathered}
$$

After calculations using the formula (6) for possible options of model saturation with information. Calculation results are provided in table 1.

Table 1 Calculation results using formula (6) for numerical example

\begin{tabular}{ccccc}
\hline No. & Operations sequence & Stages count & $\mathrm{H}$, bit & $\Phi$ \\
\hline 1 & $(\mathrm{P} 1+\mathrm{P} 2+\mathrm{P} 3+\mathrm{P} 4)$ & 1 & 1.417 & 0.292 \\
2 & $\mathrm{P} 1+(\mathrm{P} 2+\mathrm{P} 3+\mathrm{P} 4)$ & 2 & 1.801 & 0.100 \\
3 & $\mathrm{P} 3+(\mathrm{P} 1+\mathrm{P} 2+\mathrm{P} 4)$ & 2 & 1.397 & 0.302 \\
4 & $\mathrm{P} 2+(\mathrm{P} 1+\mathrm{P} 3+\mathrm{P} 4)$ & 2 & 1.316 & 0.327 \\
5 & $\mathrm{P} 4+(\mathrm{P} 1+\mathrm{P} 2+\mathrm{P} 3)$ & 2 & 1.417 & 0.292 \\
6 & $(\mathrm{P} 1+\mathrm{P} 2)+(\mathrm{P} 3+\mathrm{P} 4)$ & 3 & 1.703 & 0.149 \\
7 & $(\mathrm{P} 1+\mathrm{P} 4)+(\mathrm{P} 3+\mathrm{P} 2)$ & 3 & 1.281 & 0.360 \\
8 & $(\mathrm{P} 1+\mathrm{P} 3)+(\mathrm{P} 2+\mathrm{P} 4)$ & 3 & 1.279 & 0.361 \\
9 & {$[(\mathrm{P} 1+\mathrm{P} 2)+\mathrm{P} 3]+\mathrm{P} 4$} & 3 & 1.134 & 0.433 \\
10 & {$[(\mathrm{P} 1+\mathrm{P} 3)+\mathrm{P} 2]+\mathrm{P} 4$} & 3 & 1.246 & 0.377 \\
11 & {$[(\mathrm{P} 1+\mathrm{P} 4)+\mathrm{P} 3]+\mathrm{P} 2$} & 3 & 1.197 & 0.402 \\
12 & {$[(\mathrm{P} 1+\mathrm{P} 2)+\mathrm{P} 4]+\mathrm{P} 3$} & 3 & 1.354 & 0.323
\end{tabular}

From the calculations, we obtain the optimal (from the viewpoint of the quality of the model saturation with information) option (option No. 2). 
Thus, an objective criterion has been proposed for the information model quality formalizable for general case. The use of the described criterion allows to formalize the information management rules in the city scale and area scale distributed and grid systems.

Along with the information model compilation efficiency criterion, for the construction site information modeling management system the model data topicality criterion is also important. Peculiarity of the data topicality determination in the building information system is the continuous change of the modeled system (building) parameters.

As a model data topicality criterion, it is proposed to use the model aging vector (7).

$$
\bar{S}=\bar{R}-\bar{U}
$$

The vector is the difference between the model data update speed vectors (by data types) (8):

$$
\bar{R}=\operatorname{grad}\left(\frac{\partial I}{\partial P_{1}} ; \frac{\partial I}{\partial P_{2}} ; \frac{\partial I}{\partial P_{3}} ; \ldots \frac{\partial I}{\partial P_{n}} ;\right)
$$

and the modeling object data change rate (9):

$$
\bar{U}=\operatorname{grad}\left(\frac{\partial J}{\partial P_{1}} ; \frac{\partial J}{\partial P_{2}} ; \frac{\partial J}{\partial P_{3}} ; \ldots \frac{\partial J}{\partial P_{n}} ;\right)
$$

Thus, when the criterion (7) reaches the predefined (by the rules of the specific modeling process) value, it is necessary to start the information model update procedure. Herewith, the update plan is compiled in an optimal manner (using the information model quality criterion).

\section{Conclusion}

Thus, it appears that a group of criteria has been proposed that ensure analytical support of the construction facilities information modeling management process.

This work was financially supported by Ministry of Education and Science of the Russian Federation (7.6932.2017/8.9). All tests were carried out using research equipment of The Head Regional Shared Research Facilities of the Moscow State University of Civil Engineering (RFMEFI59317X0006).

\section{References}

1. Volkov A., Shilova, L. Some criteria of critical infrastructures stability J. MATEC Web of Conferences, 05009, 86 (2016).

2. Volkov A., Shilova, L. Principles of Formation of Stability of Construction Projects J. Procedia Engineering, 844-849. 153 (2016).

3. Volkov A.A., Vasilkin, A. Optimal Design of the Steel Structure by the Sequence of Partial Optimization J. Procedia Engineering, 850-855. 153 (2016).

4. Volkov A., Kuzina, O. Complementary Assets in the Methodology of Implementation Unified Information Model of the City Environment Project Life Cycle J. Procedia Engineering, 838-843. 153 (2016).

5. Volkov A.A., Roytman, V.M., Shilova, L.A. Model of stability of life support systems in emergency situations International Journal of Applied Engineering Research, 1666-

1669. 11 (3), (2016). 
6. Bulgakov A., Volkov, A., Sayfeddine, D. Mathematical representation of haptic robotic realization for artefacts maintenance ISARC 2016 - 33rd International Symposium on Automation and Robotics in Construction, 98-104. (2016).

7. Volkov A.A., Batov, E.I. Dynamic extension of Building Information Model for "smart" buildings. Procedia Engineering, 849-852. 111, (2015)

8. Volkov, , P. Chelyshkov, , Y. Grossman, A. Khromenkova, J. IOP Conference Series: Earth and Environmental Science, 90, 1 (2017) DOI: 10.1088/1755-1315/90/1/012203

9. Volkov, P. Chelyshkov, D. Lysenko, J. Proc. Engin. 833-837, 153 (2016) DOI: $10.1016 /$ j.proeng.2016.08.251

10. Volkov, A. Sedov, P. Chelyshkov, A. Doroshenko, J. Applied Mechanics and Materials 3231-3233, 580-583 (2014) DOI: 10.4028/www.scientific.net/AMM.580-583.3231

11. Volkov, A. Sedov, P. Chelyshkov, E. Kulikova, J. Applied Mechanics and Materials 757-760, 584-586 (2014) DOI: 10.4028/www.scientific.net/AMM.584-586.757

12. Doroshenko, J. MATEC Web of Conferences 05013, 112 (2017)

DOI: $10.1051 /$ matecconf/201711205013 